\title{
The City as an Art Integration Space
}

\author{
Mikhail Dutsev ${ }^{1, *}$
}

\author{
${ }^{1}$ Department of Architectural Environment Design, Nizhny Novgorod State University of Architecture and Civil \\ Engineering, Niznij Novgorod, Russia \\ *Corresponding author. E-mail: nn2222@bk.ru
}

\begin{abstract}
The author introduces an advanced understanding of artistic dimension in architecture and in urban environment by referring to the concept of integrity. It is suggested to use the author's method of artistic integration to consider the architectural practice in interconnectedness of historical background, cultural patterns and flexible architectural-artistic appearances, including personal, social and cultural phenomena.

The art field of interaction is defined at the intersection of three strong trends in architecture: conceptualization, theatricalization and design. It is noted that despite all disadvantages that exist in this professional field today, such as commercial and consumer orientation in architecture activities, there is a necessary turn to the creative rethinking and remaking of space.
\end{abstract}

All these motives make it possible to work at the borders of disciplines, to build new connections, to increase the potential of renewed urban space: the dialogue of times and communication, theatricalization of architecture, social activities, natural landmarks, art intervention.

Keywords: urban environment, architecture, design, contemporary art, concept, social communication, artistic integration

\section{INTRODUCTION}

How many dimensions does a modern urban space have? Does the environment of a modern city "work" as an artistic phenomenon? If yes, then what are the mechanisms of this work?

Traditionally, the compliance with the criteria of "beauty" is intuitionally expected, first of all, from the historic places formed in the course of over-placement of epochs. We seem to pay homage to the genius of the past as a kind of wise creative force: an individual master, the efforts of the ancestors, time as such. Exactly wise, but not always creative, because time destroys, and sometimes destroys irrevocably. However, even the noble appearance of ruins inspires awe and gives birth to inspiration...

Creating and developing spaces of his city, correlating their meaning with current socio-economic priorities, a person sometimes leaves out a whole range

*Fund: The reported study was funded by Science and Technology Development State Program of the Russian Federation for years 2013-2020, Program of Fundamental Research of State Academies of Science for years 2013 - 2020, within Program of Fundamental Researches of Ministry of Construction, Housing and Utilities of the Russian Federation and Russian Academy of Architecture and Construction Sciences, the Research Project 1.4.8 of meanings and motives that address the spiritual essence of the place. Perhaps, first, this happens unintentionally, and the reason can be very simple: all of us initially believe in the inviolability of the environment, the city as an "eternal" chronotope, an archetype, as an entity being stronger than we are, that has been existing before us, and will be existing after us. However, today an increasing concern about the correlation of these forces appears more often. Man has become stronger than space. Yes or no? This is a statement and a question at the same time. Probably, the man has become stronger than the "historical city", its once-chamber scale, character, and flow of time. Here we mean the very essence of the old city, its trace in our genetic memory as a synthesis of a number of archetypal meanings, a complete integral image. Then, there is a further anxiety: does this imaginary "force" make us weaker? In the scale of human evolution? We seem to lose important connections (relationships), break the currents that flow from afar... We lose dimensions, impoverishing consciousness.

As it often happens in history, the problems themselves suggest probable solutions. Today's crosssection of architectural trends shows a number of versions of extension or restoration of connections, which are equally necessary for harmony in the professional consciousness and in the surrounding 
space. At the same time, there is a sense of a lack of some important grounds: substantive and formal.

New urbanism offers to respond to the functional needs of different layers of society, to build scenarios of processes, but they often become a tribute to the momentary fashion. The increase of the urban space logistics also follows the patterns of economic development or even certain purely "mechanical" extension of management models. Consequently, socioeconomic planning or a popular spontaneous passion that has temporarily taken hold of a group of people does not provide a humane space for a person. Environmental awareness and a sustainable development strategy can be perceived as valuable guidelines that theoretically take into account cultural, natural-humanistic and technological aspects of the modern and even future world, but the aspect of artistic values in this perspective looks "optional". The growing wave of digital communications of various kinds can hardly be considered the only reliable panacea for cultural field replenishment. While recognizing the undeniable progress in informing recipients, including in aesthetic matters, and the strong interactive potential, we should also express concern about the tendency for a person to be detached from physical reality, a kind of "space of absence", which a virtual world is already becoming.

\section{A METHOD OF ARTISTIC INTEGRATION}

The question of artistic-figurative and emotionalexpressive essence of architecture has been raised for a long time, and it finds many-sided answers. Today, thinking about changing aesthetic paradigms goes to the front line of professional self-reflection. Thus, I.A. Dobritsyna in her article "Aggression and protection: to the problem of aesthetics of "strangeness" in architecture" addresses the aspect of the modern world representation, including in an important perspective: "... where and in what is the human dimension expressed now?" [1]. The researcher bases on the scientific assumption of a modern shift in priorities: "from the aesthetics of the Beautiful to the aesthetics of the Sublime". The latter contains the most extraordinary manifestations of current culture, probably written about by J.-F. Lyotard in the essay "The Sublime and the Avant-garde": "Art does not imitate nature, it creates its own world next to it ...in which the monstrous and formless has the right to be, since it can be Sublime" [2]. Here, I.A. Dobritsyna addresses another researcher, American philosopher Fredric Jameson and his concept of cognitive cartography: "Cognitive cartography aims to find ways to express the structure of the Imaginary" [3].

The "imaginary" is another side of the theme, connected here with a kind of collective imagination of society. Let's remember that the theories of psychoanalysis have opened up a wide range of paths of the unconscious, its painful, frightening manifestations. However awkward it might be to mention the "dark sides" of human consciousness, today's angle of the topic discussion requires it, especially since it is extremely difficult to draw the lines of "healthy" or "ill" art. This division may be too tendentious, biased by one of the parties. It is not by chance that I.A. Dobritsyna singles out the digital mode of architectural creativity. Architectural imagination is fully a part of the complex process of self-determination of a person, his involvement in virtuality, in the global market, in total design or ecological utopias. "The aesthetic dominant under the new system (power and society) is no longer literary or even verbal, it is represented by a visual culture, a culture of images and visual simulators. It is clear that the role of architecture is immense" [4], - this is another quote from F. Jameson, indirectly emphasizing the figurative side of the profession. Of course, here, visuality is both a statement and a question, and even a challenge. After all, there may be a very effective substitution of reality, which sometimes occurs...

In order to reconcile many of today's disputes and disagreements regarding the criteria of the Beauty and art, it is proposed, first of all, to change the categorical apparatus and the principle of measure, starting not from the concept of "beauty", but from the concept of "integrity". Integrity expresses the deep correlation and unity of the part and the whole, inherent in the creations of nature itself and the highest achievements of man outstanding Works of Art. The principle of artistic integrity itself helps to correlate the qualities and properties of space with categories of a higher order: an object with the town; subjective time with evolutionary processes, the total flow of time; individual values and memory of locations; artistic techniques with aesthetic tradition; the author's approach of an architect with trends, views and appraisals of contemporaries, including professional and nonprofessional judgments, and emotional responses.

Today the current Internet space poses even more questions about collective or individual modes of reading information. According to the theorists of Metamodernism, for example, A. Cauquelin, the network implements "technoimages" [5] as fruits of free information exchange. Leading architects, such as T. Mayne, write about possible forms of collective authorship. Similar positions are made public by $\mathrm{C}$. Jencks in the "New paradigm in architecture", stating that information becomes a creative tool, and architecture, in a certain sense, is depersonalized. According to the researcher, datascapes form "arrays of data based on various hypotheses", which "allow the computer to model independently around each of the hypotheses its possible spatial embodiments" [6]. 
All these are features of the world in the era of globalization or, more precisely, (what I want to believe), the world with prevailing global and growing local trends. The first is, perhaps, the indisputable attribute of reality, and the second is a desired compensating component. In the Internet an individual user easily chooses his favourite authors, subscribes to interesting newsletters and blogs. In this situation, it is difficult to talk about the opinion of a respected expert community, since a person is more than ever absolutely free to ignore it by following a random link. This situation radically changes the system of "quality control" and the hierarchy of levels of cultural phenomena. The field of today's values is by nature frighteningly non-hierarchical.

Is the architecture an art?

Architecture has always been and continues to be an art, as well as philosophy, engineering, sociology, and ecology - all these should be again related to "free sciences and arts" in an updated format of understanding. Recognizing that such a liberty may face a series of protests that are largely justified, we once again suggest to refrain from unambiguous definitions. Yet again, let's focus on the integrity. It is then that we come to a different understanding of the architect-artist. Probably, this is such a creative personality who generates and (or) interprets integral entities (spatial, intellectual, emotional, general structural) that go back to the completion of the author's statement and are included in environmental contexts.

So, the concepts of "integrity", "connectedness" (connection), "field" and "integration" become the basis of the proposed methodology. The latter, perhaps, is the most "heavy" on sounding, and in addition, has come from the near-economic fields of knowledge; however, it is important as a designation of the process of moving to the desired integrity. The "artistic" can be omitted or used conditionally as an indication of the corresponding figurative and expressive side of architecture, which has undergone such a significant transformation. It is important that the proposed concept of connectedness is fundamentally broader than the aesthetic sphere, and extends far beyond it.

The field as a place and as a tool contains areas of values that are dynamically mixed in a constant stream, or rather a vortex. Spatial and temporal patterns, artistic and plastic languages (language codes of old and new arts), personal worlds of architects, and various emerging trends in related fields of activity interact and overlap in various ways in this vortex. There is in many ways controversial question of hierarchy: do the masters' holistic approaches crown this system, and are the fields expressed through the prism of the author's creativity? It is intuitive to assign this model a nonhierarchical character of absolute freedom, when priorities are unpredictable every time. On the other hand, at this historical moment of "architectural pluralism" (according to the well-known definition of A.V. Ikonnikov), it is more convenient and reliable to rely exactly on the master's individual "style" expressed in his best objects. From this point of view, today's architectural world is a field of masters, their concepts, and their deliberate or instinctive choice.

Thus, the field model of architectural and "artistic" integrities is timely and transparent. Only the mechanism of using this model for building the usual hierarchy of values remains a fundamentally unresolved issue. Probably, finding the criteria in a dynamic system becomes as relative and temporary, as constantly changing values in life and creativity are.

\section{VISION OF SPACE AND ARCHITECTURAL INTEGRITY}

The current state of fragmentation, dispersion of semantic meanings, professional guidelines and artistic criteria raises the question of integrity in a new way. As it has already been noted, this concept is able to return the complementarity of the system on the basis of the laws of universal connectivity of all phenomena of the world, and inclusion of a person in this circle. The desired conceptual structure makes the meeting of the old and the new, the natural and the man-made, the individual and the mass, the author's and the unknown, the singular and general consistent - they coexist seamlessly. A productive reconciliation that is yet difficult to imagine in reality. It is no accident that the holistic view has a long tradition in almost all branches of spiritual practices, in art, philosophy, and, in a certain sense, in science (rather, the humanities, intuitive insights, and interdisciplinary cycles).

Frank Lloyd Wright described the desired unity in his architectural concept: "Organic" means essential, intrinsic, inherent in something, integrity in the philosophical sense, where the whole is so related to the part, as the part to the whole, and where the nature of the materials, the nature of the purpose, the nature of everything, being implemented, becomes clear, acting as a necessity. It follows from this nature what character a true artist can give to a building in these specific conditions" [7]. In this quote, there is no direct reference to "organic architecture" - the author seems to rise above the subject of professional activity and conducts discussion on a more general level, where the organic takes on the meaning of integrity.

This logic also includes the form category, which is traditionally quite important in architecture. Louis Henry Sullivan wrote: "Form exists in everything, everywhere, and in every moment... some forms are defined, others are uncertain; some have symmetry, others only rhythm. Some are abstract, others are material. Some attract sight, others hearing, touch, or 
any combination of them. But all forms unmistakably symbolize the connection between the material and the immaterial... between the boundless spirit and the limited mind" [8]. Here the law of form is indicated, which always has a dual nature, telling in the language of physical reality about the invisible mysteries of the world.

Creative individuality during different periods of life and at the moment of creativity is marked, probably, by the most intense and non-linear mode of integrity. Flickering, quantum algorithms that are not identical to the "creativity" of nature are more suitable for describing it. Thus, Finnish theorist Juhani Pallasmaa writes that "the artist's attention is always simultaneously on two main poles: the external world and his own personality, and, as a consequence, any creative work is at the same time a micromodel of the cosmos, and an unconscious self-portrait" [9]. Probably, the most important integrative basis of the architect's work, as an artist in the broad sense of the word, is expressed in the deep personal conditioning of reality.

\section{ART FIELD OF THE MODERN ARCHITECTURE}

The actual context of convergence and mutual germination of architecture and the entire field of art (artistic and creative activity) is associated with the active development of basic trends that can be generically defined as "theatre" and "design", together with the conceptualization of creativity, as well as the strengthening of the universal natural-humanistic foundation of architectural activity. In the mentioned actual spheres of architectural and artistic integration, the figurative principle is involved both in the traditional, "tangible" sense of working with form, and in a more abstract form - at the level of consciousness, sensory perception and the feeling of space. These centres combine the most popular integrative areas of architectural art: conceptuality, theatricality, entertainment, scenarionism, media, illusory, elemental art, digitality, and virtuality [10]

The trend of theatricalization is based on the accent form and scenario involvement of the addressee in the architectural performance. Therefore, different kinds of principles are required here. First, the emotional and plastic principle of the work with a three-dimensional or spatial form is accompanied by an interpretation of the sculptural qualities of the work. Second, the social principles are used to form interactive qualities of architecture: game techniques, navigation, recreation, transformation and mediation. A socially activated space is obviously not only artistic, but is focused on comfort, accessibility, and commercial success. These groups of principles are closely intertwined, determining the methods of scenario organization of movement and self-presentation, symbolic and archetypal forms of "speaking" architecture, creating an illusory image and aesthetics of a miracle. The choice of material that complicates reality, such as glass, mirrors, and fabrics, is also made in the same way.

Today the "artistic" approach to design supposes several provocative characteristics - going beyond the typological framework, the boundaries of scale, and the integrity of the context. The formation of the architectural environment design can take place on a different level of scale, starting from the person, the trajectory of his movements in space, emotional plots and moods. When viewed "from the inside", the artistic image does not require functional conditioning to a certain extent. Compositional characteristics that traditionally originate in the scale of the general layout can now be replaced by local implementation of the design code on all scale levels. Exactly this design code, which carries both imaginative and playful features, as well as advertising ones, should be considered the epicentre of the plastic foundations of the concept of "design". On the other hand, architectural "design" requires creation of comfort and a certain logistic scheme based more on social research and management.

The plastic binding of the conceptual basis is not so obvious, but it should be considered from the point of view of integrity. We take this foundation as an area of architecture's borrowing the meanings of contemporary art, a kind of new positioning of architectural creativity. Recognizable techniques of conceptual experiments can move into space, be used in the environment from landscaping to the building. At the same time the conceptual perspective itself has a long tradition in architecture, and has developed certain canons: addressing history or future (G. B. Piranesi, utopias and retro-futurism), returning to the ideals of nature (the Garden City of E. Howard, designs of Soviet urbanists and desurbanists), striving for clear geometric constructions (É.-L. Boullée, C. N. Ledoux, I. Leonidov), dissolution in metaphysical fields and in pure art (classic of the genre Ya. Chernikhov, domestic "portmanteau" Yu. Avvakumov, A. Bavykin, M. Belov, A. Brodsky, I. Utkin, M. Filippov, M. Khazanov, S. Choban, artist of architectural anti-utopias L. Woods), technocratic and ecological concepts (the Archigram group, Japanese conceptualists, T. Mayne), architectural theories and manifests (A. Sant'Elia, W. Gropius, F.L. Wright, Le Corbusier, A. Aalto, K. Kurokawa, O.-M. Ungers, R. Koolhaas, S. Hall, P. Zumthor, P. Shumakher, Ju. Pallasmaa, A. Rappaport and others).

The natural-humanistic principle addresses the interpretation of natural motives and phenomena, integration into the natural context, and integral experiences of the unified spatial body of nature and 
man. At the same time, the less literally this unity is understood, the more abstract the plastic language is, which seeks to use not the external similarity, but the fundamental laws of harmonious coexistence. Today, the direct integration of natural factors into architecture, in conceptual, artistic, and constructive-technological directions, is also relevant. Architectural bionics, biomimetic principles, biometric structures, "green" architecture, and land forming, - areas interpreting nature metaphorically, structurally, or using natural components as the material of space, have firmly become part of the arsenal of the modern architect. "The art of the elements" has become an object of architectural plasticity, as if completing the circle of preferences of the modern world: from the desire for the play and the stage to the attempt to return to natural beauty.

\section{CREATIVE RESOURCE OF THE SPACE OF CRISIS PERIOD}

Today's society is characterized by a constant experience of crises associated with the action of consumer society mechanisms. We can assume that the modern man is constantly immersed in the space of "crisis period". This is a physically lived-in urban space or other environment, as well as mental projections and images of space in the insight of the individual. This state is marked by a number of features, which include a constant change of priorities and rapidly coming "fatigue" from previous ideals; creative, including collective, forms of leisure; strong, often defining market influences. First of all, we are interested in those features that allow us to reveal the potential of the space in its actual values. It is necessary to emphasize two directions in the interpretation of spaces - the path of spatial "co-creation" with the man.

Space as a mediator, interlocutor, medium: the space of participation - co-creation with the user; dematerialized space - blurring material boundaries and embodiments; virtual space - the space after and "outside" itself; media space — broadcast and interactive.

Another important resource is the action of borderline social, natural, and artistic-spatial forms of the environment.

Border space resource: the space of social action; the space of a sign and a symbol; phenomenological space; ecological space; artistically interpreted space; performative space - play, scene; mediative and virtual space; metaphysical space (memory, imagination, mythology, vision); mixed integral space; effect of absence - the "empty" space of free opportunity.

Note that these two groups overlap in many ways and often act as a single creative resource, which, of course, does not remove the actual contradictions, but rather reveals their creative sides and allows the integrative potential and new aesthetics of the environment to be realized.

\section{CONCLUSION}

Summing up our considerations, the analysis of examples and directions of architectural thought, we still cannot free ourselves from the feeling of anxiety and uncertainty. Does architecture today belong to the circle of arts that is interesting and understandable to society from the artistic viewpoint? Most likely, the answer to this question will be negative..

Probably, the current trend of transferring some of the architect's works into areas, that border on design and contemporary art, indicates the need for special communication with the addressee beyond strict disciplinary "boundaries". Similar processes towards urban spaces and intellectual resources of communication occur globally in all artistic culture. Not by chance, that Situationists have also proposed and developed a line of modelling situations and scenarios, and the artist has been more of a creator of meanings rather than images, manifestos, and not aesthetic works, for more than a decade.

On the one hand, this shows a "flight" from the foundations of the profession, its crisis is visible. Let us recall the publications of A.G. Rappaport and many disappointing conclusions that we have to agree with. Architecture, indeed, has lost a lot and continues to lose in its craft, language, traditional quality criteria - in all, that can be called cultivated and evolving over the centuries "professional culture", in some of its "privilege".

Nevertheless, contemporary world gives us certain encouraging signs: the moment of accustoming of a person to architecture in the context of communication, engagement and entertainment is coming. It is possible that these new hybrid forms are not truly architectural, but they help address some of the original architectural motifs to our contemporary. Particularly performative, interactive, and festival practices, which are able to gather a large interested audience and interact with it, help architecture become open and accessible to people.

We accept that these strategies are part of the system of consumer society, and the question of how much creativity is able to preserve the quality of high art in such conditions, remains open. Today we can observe, explore, and participate in these borderline creative manifestations, hoping for a revival of the integral architectural and artistic space of communication of the modern city. 


\section{References}

[1] I.A. Dobritsyna, Agression and defence: to the problem of aesthetics of "strangeness" in architecture // Issues of the theory of architecture. Architecture: contemporary experience of professional self-reflection. Proceedings of the IX and $\mathrm{X}$ Ikonnikov readings / ed. by I.A Dobritsyna. Moscow: LENAND, 2017, pp. 144-164. [in Russian].

[2] I.A. Dobritsyna, Architecture of the XXI century. Problems of representation of reality // Issues of the theory of architecture. Architecture: contemporary experience of professional selfreflection. Proceedings of the IX and X Ikonnikov readings. Ed. By I.A Dobritsyna. Moscow: LENAND, 2017, p. 47. [in Russian]

[3] Ibid.

[4] Ibid.

[5] O.A. Mitroshenkov, What will replace postmodernism? // Free thought (Svobodnaya mysl). 2013. No 3. URL : http://svom.info/entry/355-chto-pridet-na-smenupostmodernizmu/ (accessed: 26.02.2020) [in Russian]

[6] C.A. Jencks, The new paradigm in architecture // Proekt international 5. 2003. Vol. 5. URL: http://cih.ru/ae/ad37.html (accessed: 26.02.2020) [in Russian]

[7] F.L. Wright, The Future of Architecture, Moscow: Gosizdat lit. po str-vu, arkhitekture i stroit. materialam Publ., 1960, p. 33. [in Russian]

[8] As cited in: D.O. Shvidkovskiy, From megalith to megalopolis: essays on the history of architecture and urban planning, Moscow: Arkhitektura-S Publ., 2009, pp. 14-15. [in Russian]

[9] Yu. Pallasmaa, The Thinking Hand: Essential and Embodied Wisdom in Architecture, Moscow: Klassika-XXI Publ., 2013, p. 129. [in Russian]

[10] M.V. Dutsev, The concept of art integration in contemporary architecture, Nizhny Novgorod: NNGASU, 2013, 388 p., ill. [in Russian] 